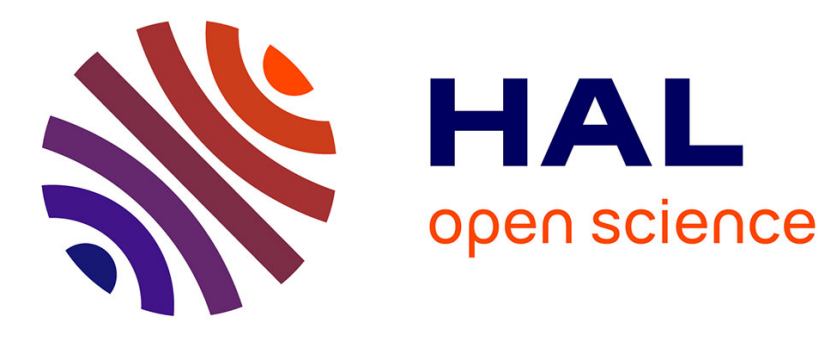

\title{
Scaling of correlation functions near capillary condensation
}

\author{
Andrew Parry, Nelson Rei Bernardino, Carlos Rascón
}

\section{To cite this version:}

Andrew Parry, Nelson Rei Bernardino, Carlos Rascón. Scaling of correlation functions near capillary condensation. Molecular Physics, 2011, pp.1. 10.1080/00268976.2010.538739 . hal-00686159

\section{HAL Id: hal-00686159 \\ https://hal.science/hal-00686159}

Submitted on 8 Apr 2012

HAL is a multi-disciplinary open access archive for the deposit and dissemination of scientific research documents, whether they are published or not. The documents may come from teaching and research institutions in France or abroad, or from public or private research centers.
L'archive ouverte pluridisciplinaire HAL, est destinée au dépôt et à la diffusion de documents scientifiques de niveau recherche, publiés ou non, émanant des établissements d'enseignement et de recherche français ou étrangers, des laboratoires publics ou privés. 


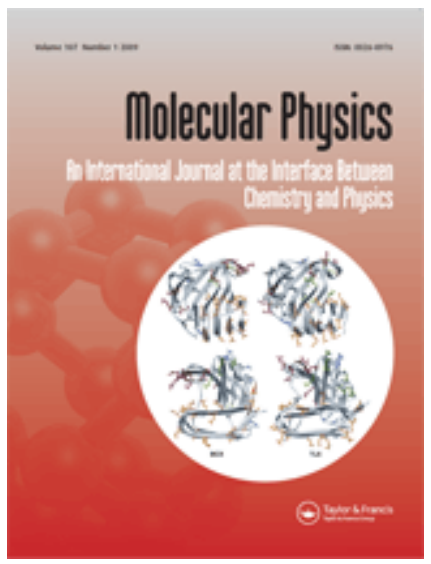

\section{Scaling of correlation functions near capillary condensation}

\begin{tabular}{|c|c|}
\hline Journal: & Molecular Physics \\
\hline Manuscript ID: & TMPH-2010-0363.R1 \\
\hline Manuscript Type: & Special Issue paper - In honour of Bob Evans \\
\hline $\begin{array}{l}\text { Date Submitted by the } \\
\text { Author: }\end{array}$ & 22-Oct-2010 \\
\hline Complete List of Authors: & $\begin{array}{l}\text { Parry, Andrew; Imperial College London } \\
\text { Bernardino, Nelson; Max-Planck-Institut für Metallforschung } \\
\text { Rascón, Carlos; Universidad Carlos III de Madrid, Mathematics }\end{array}$ \\
\hline Keywords: & $\begin{array}{l}\text { Capillary Condensation, Wetting, Correlation Function, Effective } \\
\text { Hamiltonian, Scaling }\end{array}$ \\
\hline \multicolumn{2}{|c|}{$\begin{array}{l}\text { Note: The following files were submitted by the author for peer review, but cannot be converted } \\
\text { to PDF. You must view these files (e.g. movies) online. }\end{array}$} \\
\hline $\begin{array}{l}\text { source files.zip } \\
\text { capillary.tex } \\
\text { wetting.bib }\end{array}$ & \\
\hline
\end{tabular}

\section{SCHOLARONE" Manuscripts}




\title{
Scaling of correlation functions near capillary condensation
}

\author{
Andrew O. Parry \\ Department of Mathematics, Imperial College London, London SW7 2BZ, UK \\ Nelson R. Bernardino \\ Centro de Física Teórica e Computacional, Avenida Professor Gama Pinto 2, P-1649-003 Lisbon, Portugal and \\ Departamento de Física, Faculdade de Ciências da Universidade de Lisboa, P-1749-016 Lisbon, Portugal \\ Carlos Rascón \\ Grupo Interdisciplinar de Sistemas Complejos (GISC), Departamento de Matemáticas, \\ Universidad Carlos III de Madrid, 28911 Leganés, Madrid, Spain
}

\begin{abstract}
We study the influence of wetting films on (density-density) correlations for a fluid in a slit-like geometry near capillary condensation. We show that, for systems with short-ranged forces, the interaction between the wetting films strongly enhances the amplitude of the exponential decay of correlations and, unlike the interfacial roughness, is independent of a high-momentum cut-off. The correlation function shows scaling behaviour near condensation arising from the equality of two characteristic length scales: the parallel correlation length (associated with the complete wetting films) and a length scale related to the non-local interaction between the wetting films on either side of the slit. We introduce a dimensionless amplitude ratio associated with the decay of correlations which allows us to distinguish between local and non-local effective Hamiltonian theories. Only the latter is fully consistent with microscopic density functional descriptions of correlation functions. The influence of long-ranged intermolecular forces and fluctuation effects in two dimensions is also discussed.
\end{abstract}

\section{INTRODUCTION}

Capillary condensation [1-7] refers to the shift in the value of the chemical potential $\mu_{\text {cap }}$ (or pressure), relative to that of bulk saturation $\mu_{\text {sat }}$, at which a vapour confined in a slit of width $L$ condenses to a liquid-like phase, see Fig. 1. As is well known, for macroscopically wide slits, the shift in the coexistence curve, $\delta \mu_{\text {cap }} \equiv \mu_{\text {sat }}-\mu_{\text {cap }}$, is well described by the classical Kelvin equation

$$
\delta \mu_{\text {cap }}=\frac{2 \sigma \cos \theta}{\Delta \rho L}+\cdots
$$

where $\theta$ is the contact angle, $\sigma$ is the surface tension of the liquid/vapour interface and $\Delta \rho=\rho_{l}-\rho_{v}$ is the difference in bulk number density. For thin-film uni-axial (Ising-like) magnets there is an analogous shift in the value of the external field $h$, at which up-spin and down-spin-like phases coexist $[6,8]$. In this magnetic notation, "condensation" between phases with net positive and negative magnetisation occurs when $h_{\text {cap }} \approx-\sigma \cos \theta / m_{0} L$ where $m_{0}$ is the spontaneous magnetisation and $\sigma$ is the surface tension of the free up-spin/down-spin interface.

Modern density functional theory (DFT) studies and computer simulations have shown that, at low temperatures, far from the capillary critical point, the Kelvin equation remains accurate for slit widths as small as 10 molecular diameters provided the walls are only partially wet by the liquid, corresponding to $\theta>0$. For complete wetting, $\theta=0$, (or indeed complete drying $\theta=\pi$ ) on the other hand corrections to the Kelvin equation arise due to the presence of wetting layers of liquid (vapour for the case of drying) at each wall and are particularly important for systems with long-ranged (dispersion-like) interactions in the wetting layers [9]. Similar (fluctuation-induced) modifications to the Kelvin equation arise in lower dimensions due to the long-ranged entropic repulsion of the interface from the wall(s) [10].

In this paper, we point out that, for systems with shortranged forces, complete wetting layers also strongly influence the decay of (density-density) correlations near capillary condensation. While there has been some discussion of the moments of the correlation function and, in particular, their relation to thermodynamic observables such as the force of solvation $[1,11]$, we are not aware of any detailed studies of how wetting influences the asymptotic decay of the correlation functions in the capillary slit geometry. We ask the following question: what is the correlation (function) between particles situated on opposite side of the capillary? On the "condensed" side of the capillary condensation transition, $\mu=\mu_{\text {cap }}^{+}$, it is reasonable to assume the correlation function across the slit is similar to that of a bulk liquid. On the "vapour" side of the transition, $\mu=\mu_{\text {cap }}^{-}$, however it is possible that the correlation is enhanced due to the interaction between the fluctuating liquid-vapour interfaces associated with the wetting films. We shall show that the full quantitative form of this enhancement is particularly revealing and reflects the non-local nature of interfacial interactions. Indeed, this leads to universal scaling behaviour and a novel reinterpretation of the Kelvin equation.

Our article is arranged as follows: we begin by recalling some simple background theory about complete wetting transitions and the Kelvin equation. We then turn attention to the nature of correlation functions, and use effective interfacial Hamiltonian descriptions to predict how the spatial decay across the capillary depends on the slit width. We focus primarily on three-dimensions (3D) in systems with shortranged forces, and stress some subtle quantitative differences between predictions based on a local and non-local description of interfacial phenomena. Only the latter is fully consistent with calculations based on a more microscopic squaregradient density-functional theory. We finish with a summary 


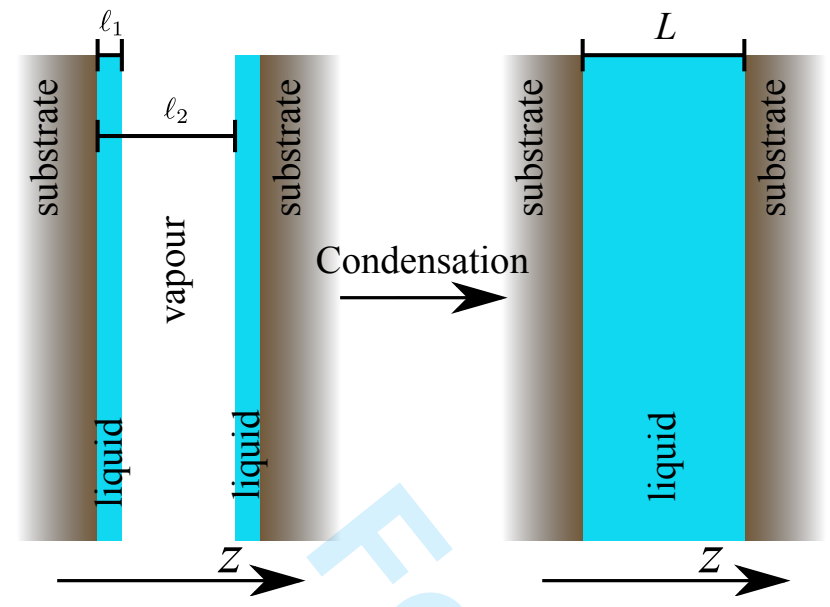

FIG. 1. Schematic illustration of coexisting vapour-like and liquidlike phases in a capillary slit geometry of width $L$. On the vapour side of the condensation transition, thick wetting films of liquid form at the substrates if the contact angle is zero.

of our main predictions and also make some remarks about the decay of correlations in 3D with long-ranged (dispersion) forces, and also in 2D concentrating on Ising-like systems.

\section{BACKGROUND THEORY}

Consider the interface between a planar substrate (wall) of infinite area and a bulk fluid at chemical potential $\mu$ and temperature $T$, below that of bulk criticality. If $\mu>\mu_{\text {sat }}$, the bulk fluid is a high density liquid while for $\mu<\mu_{\text {sat }}$ it is a low density gas. Exactly at bulk two-phase co-existence the surface tensions of the respective wall-vapour $\left(\sigma_{\mathrm{wv}}\right)$ and wall-liquid $\left(\sigma_{\mathrm{wl}}\right)$ interfaces are related through Young's equation for the contact angle of a macroscopic drop:

$$
\sigma_{\mathrm{wv}}=\sigma_{\mathrm{wl}}+\sigma \cos \theta .
$$

Complete wetting corresponds to $\theta=0$, in which case a macroscopically thick layer of liquid intervenes between the wall and bulk vapour when $\mu=\mu_{\text {sat }}^{-}$[12-15]. The complete wetting transition refers to the continuous divergence of the thickness $\ell_{\pi} \sim \delta \mu^{-\beta_{s}^{\text {co }}}$ of this layer as saturation is approached $\left(\delta \mu=\mu_{\mathrm{sat}}-\mu \rightarrow 0\right)$. Here, the subscript on the thickness refers to the semi-infinite nature of the planar wall-fluid geometry. Associated with this transition are the vanishing of a singular contribution to the (wall-vapour) surface tension, $\sigma_{\text {sing }} \sim \delta \mu^{2-\alpha_{s}^{c o}}$, and the divergence of a correlation length parallel to the unbinding liquid-vapour interface, $\xi_{\|} \sim \delta \mu^{-\nu_{\|}^{\text {co }}}$ (and an associated interfacial roughness $\xi_{\perp}$ ) arising from its fluctuations. Only one of the exponents is free since $1-\alpha_{s}^{\mathrm{co}}=-\beta_{s}^{\mathrm{co}}$ and $\alpha_{s}^{\mathrm{co}}=2 \nu_{\|}^{\mathrm{co}}$ which follow from the Gibbs adsorption equation and a compressibility sum-rule respectively. The phenomenon of complete drying $(\theta=\pi)$ is completely analogous to this but instead refers to the divergence of a macroscopic layer of vapour at a wall-liquid interface as $\mu \rightarrow \mu_{\text {sat }}^{+}$.
Complete wetting transitions can also be seen in semiinfinite Ising-like magnets [16-18]. Suppose for example that the spins are subject to a bulk field $h \leq 0$, (which ensures that the bulk phase has negative magnetisation) but also that a strong positive surface field $h_{1}$ orders the spins at the surface (wall). As $h \rightarrow 0^{-}$a wetting layer of up-spins grows and intrudes between the surface and the bulk down-spin state. Provided one is above the roughening temperature (for models based on lattices) the unbinding (and associated fluctuations) of the up-spin/down-spin interface are the same as those occurring for the liquid-vapour interface in fluid systems.

The modern theory of complete wetting, which is in excellent agreement with experiment [12-15], builds on the pioneering work of Frumkin and Derjaguin in the 1940s [9, 19]. Theorists have two main approaches; Density functional models and effective interfacial Hamiltonians which each have complementary strengths (and weaknesses). Within DFT the equilibrium one-body density profile is found from minimisation of a grand-potential functional. The advantage of density functional theory is that it is microscopic, taking as its starting point a molecular description of short-ranged repulsive and long-ranged attractive intermolecular forces. Thus modern DFTs based on fundamental measure theory can describe very well packing effects which give rise to density oscillations when a high density liquid is close to a wall [20].

The disadvantage of the mean-field DFT approach is that it does not include fluctuation effects arising from the thermally induced wandering of the unbinding interface. To model these one must usually rely on interfacial Hamiltonian models which describe long wavelength fluctuations in a collectivecoordinate $\ell(\mathbf{x})$, representing the local height of the liquidvapour interface (or up-spin/down-spin interface for Ising-like magnets) at position $\mathbf{x}$ along the wall. The standard local interfacial model, which is partly phenomenological in character, has the form [14]

$$
\mathcal{H}[\ell] \equiv \int \mathrm{d} \mathbf{x}\left\{\frac{\sigma}{2}(\nabla \ell)^{2}+W(\ell)\right\}
$$

where $W(\ell)$ is the binding potential function describing the direct interaction of the interface with the wall (arising from intermolecular forces) which can be determined from some more microscopic theory - most commonly a DFT for fluids or a Landau-Ginzburg-Wilson (LGW) model for Ising-like magnets [21]. By definition, the binding potential is identified as the excess-free energy per unit area of a wetting film which is constrained to be at a uniform height $\ell$. There is therefore an implicit assumption of locality in the standard interfacial model in that the interface interacts with the wall via its local height only. This is something we will return to later. Note that in writing (3) we have specialised to isotropic fluid interfaces in which the coefficient of the gradient term (the stiffness) is identified with the surface tension. This is also appropriate for Ising-like systems close to the bulk-critical point. Thus the first term in (3) arises simply due to the increase in surface area induced by a fluctuation. Implicit in the Hamiltonian is a high-momentum cut-off $\Lambda$ of the order of an inverse bulk correlation length.

A mean-field analysis of fluctuation effects at complete 
wetting in three-dimensional systems suffices for almost all purposes, implying that the film thickness $\ell_{\pi}$ and excess freeenergy $\sigma_{\text {sing }}$ can be determined from the minimisation conditions

$$
W^{\prime}\left(\ell_{\pi}\right)=0 \quad \sigma_{\text {sing }}=W\left(\ell_{\pi}\right)
$$

respectively. The length scales $\xi_{\|}$and $\xi_{\perp}$ can be determined from the structure factor (the parallel Fourier transform of the height-height correlation)

$$
\tilde{S}(q) \equiv \int \mathrm{d} \mathbf{x}_{12}\left\langle\delta \ell\left(\mathbf{x}_{1}\right) \delta \ell\left(\mathbf{x}_{2}\right)\right\rangle e^{i \mathbf{q} \cdot \mathbf{x}_{12}}
$$

where $\delta \ell(\mathbf{x}) \equiv \ell(\mathbf{x})-\ell_{\pi}$. At mean-field, $\tilde{S}(q)=1 / \tilde{C}(q)$ where $\tilde{C}(q)$ is the Fourier transform of the "direct" correlation function $C\left(x_{12}\right) \equiv \beta \frac{\delta^{2} \mathcal{H}}{\delta \ell\left(\mathbf{x}_{1}\right) \delta \ell\left(\mathbf{x}_{2}\right)}$ where $\beta=1 / \mathrm{k}_{\mathrm{B}} T$. Clearly

$$
\tilde{C}(q)=\beta\left(W^{\prime \prime}\left(\ell_{\pi}\right)+\sigma q^{2}\right)
$$

so that

$$
\tilde{S}(q)=\frac{\mathrm{k}_{\mathrm{B}} T}{W^{\prime \prime}\left(\ell_{\pi}\right)+\sigma q^{2}}
$$

and identifies the parallel correlation length as $\xi_{\|}=$ $\sqrt{\sigma / W^{\prime \prime}\left(\ell_{\pi}\right)}$. Integrating $\tilde{S}(q)$ over all wave-vectors determines the (squared) interfacial roughness $\xi_{\perp}^{2} \equiv\left\langle\delta \ell^{2}\right\rangle$ in three dimensions as

$$
\xi_{\perp}^{2}=\frac{\mathrm{k}_{\mathrm{B}} T}{2 \pi} \int_{0}^{\Lambda} \mathrm{d} q \frac{q}{W^{\prime \prime}\left(\ell_{\pi}\right)+\sigma q^{2}}
$$

The presence of the high momentum, or short-distance, cutoff is essential to regularise this integral and leads to

$$
\xi_{\perp}^{2} \approx \frac{\mathrm{k}_{\mathrm{B}} T}{2 \pi \sigma} \ln \Lambda \xi_{\|}
$$

which diverges (weakly) as one approaches complete wetting $[22,23]$. It is this length scale that is missing in current meanfield DFT descriptions of wetting transitions.

For systems with short-ranged forces, the appropriate choice of binding potential, is

$$
W(\ell) \equiv \bar{h} \ell+a e^{-\kappa \ell}+\cdots
$$

where the coefficient of the exponential repulsion $a>0$ and $\kappa \equiv 1 / \xi_{\mathrm{b}}$ is the inverse bulk correlation length $\xi_{\mathrm{b}}$ of the phase that wets the wall. Here $\bar{h}$ is proportional to the bulk-ordering field; specifically $\bar{h}=\Delta \rho \delta \mu$ for fluids and $\bar{h}=2 m_{0}|h|$ for Ising-like magnets, where $m_{0}$ is the absolute value of the magnetization of the bulk phase. The physical origin of this binding potential will be discussed later. Using this binding potential it follows that

$$
\kappa \ell_{\pi} \sim-\ln \bar{h}
$$

and the associated free-energy singularity $\sigma_{\text {sing }} \sim-\xi_{b} \bar{h} \ln \bar{h}$. Similarly for the parallel correlation length we obtain

$$
\xi_{\|}=\sqrt{\frac{\sigma}{\kappa \bar{\gamma}}} \quad(12)
$$

The values of the critical exponents are therefore $\beta_{s}^{\text {co }}=$ $0(\ln ), \alpha_{s}^{\text {co }}=1$ and $\nu_{\|}^{\text {co }}=1 / 2$. Even though the upper critical dimension for complete wetting with short-ranged forces is $d^{*}=3$, explicit renormalisation group analysis of the interfacial Hamiltonians shows that these mean-field predictions are largely unaltered by fluctuation effects. Only the coefficient of the logarithmic divergence is (slightly) altered while the expression for $\xi_{\|}$is unchanged [24].

Let us now consider a fluid confined between two identical planar walls, of infinite area $A$, which are located in the planes $z=0$ and $z=L$ respectively, and which is also in contact with a bulk reservoir at chemical potential $\mu<\mu_{\text {sat }}$ and temperature $T$. The Kelvin equation (1) follows from consideration of the total grand potential $\Omega$ for vapour-like (v) and liquid-like (l) phases that may coexist in the slit at temperatures far below the capillary critical point [1]. For the gas-like phase we write

$$
\frac{\Omega_{v}}{A}=-p L+\sigma(L ; \mu)
$$

where $p$ is the pressure of the bulk vapour and $\sigma(L ; \mu)$ is the total surface tension of the finite-width capillary. To first approximation we write this as $\sigma(L ; \mu) \approx 2 \sigma_{\mathrm{wv}}$ corresponding to the tensions of two semi-infinite wall-gas interfaces. For the liquid-like phase on the other hand

$$
\frac{\Omega_{l}}{A} \approx-p^{*} L+2 \sigma_{\mathrm{wl}}
$$

where $p^{*}$ is the pressure of a metastable bulk liquid and $\sigma_{\mathrm{wl}}$ is the surface tension of a semi-infinite wall-liquid interface. Phase co-existence in the slit occurs when $\Omega_{l}=\Omega_{v}$ implying that $p-p^{*}=2\left(\sigma_{\mathrm{wv}}-\sigma_{\mathrm{wl}}\right) / L$. This reduces to eq. (1) on using Young's equation $\sigma_{\mathrm{wv}}=\sigma_{\mathrm{wl}}+\sigma \cos \theta$ and the bulk relation $p-p^{*}=\left(\rho_{l}-\rho_{v}\right) \delta \mu$. The Derjaguin correction to the Kelvin equation for complete wetting arises from the singular contribution to the surface tension $\sigma(L ; \mu)$ due to the wetting films. Neglecting the interaction between the wetting layers on either side of the strip we now approximate $\sigma(L ; \mu) \approx 2\left(\sigma_{\mathrm{wl}}+\sigma+\sigma_{\text {sing }}\right)$ where, as above, $\sigma_{\text {sing }} \sim \bar{h}^{2-\alpha_{s}}$. On making use of the Gibbs adsorption equation this leads to an effective reduction in the slit width $L_{\text {eff }}$ appearing in the Kelvin equation. As first shown by Derjaguin [9] for dispersion forces $L_{\text {eff }}=L-3 \ell_{\pi}$ where recall $\ell_{\pi} \approx \delta \mu^{-1 / 3}$. For short-ranged forces on the other hand the correction has a more obvious geometrical interpretation, $L_{\text {eff }}=L-2 \ell_{\pi}$, and is not quite so important owing to the slow logarithmic divergence of $\ell_{\pi}$.

\section{CORRELATION FUNCTIONS AT CAPILLARY CONDENSATION IN SYSTEMS WITH SHORT-RANGED FORCES}

\section{A. The Zeroth Moment}

Let us now begin to extend the above argument with a view to calculating the pair correlation function in three dimensions 
in systems with short-ranged forces. To do this, we will need to account for the interaction between the wetting films on either side of the slit. To this end, we write

$$
\sigma(L ; \mu)=2 \sigma_{\mathrm{wl}}+2 \sigma+W_{\text {sing }}
$$

and seek to determine the width dependence of the singular contribution to the excess-free energy. At mean-field level, which is accurate for almost all purposes in $3 \mathrm{D}$, we can determine $W_{\text {sing }}$ as the minimum value of a binding potential function which allows for the interaction of the interfaces with the walls and also each other. We write

$$
W_{L}\left(\ell_{1}, \ell_{2}\right)=W\left(\ell_{1}\right)+W\left(L-\ell_{2}\right)+\Delta W\left(\ell_{1}, \ell_{2}\right)
$$

where $\ell_{1}$ and $\ell_{2}$ denote the positions of two planar constrained liquid-vapour interfaces representing the thickness of the wetting layers (see Fig. 1). The interaction of each interface with the (closest) wall is modelled using the binding potential $W(\ell)$ defined for a semi-infinite geometry (10) while for the interface-interface interaction we use

$$
\Delta W\left(\ell_{1}, \ell_{2}\right)=-c e^{-\kappa\left(\ell_{2}-\ell_{1}\right)}+\cdots
$$

where $c \propto \sigma$ is the coefficient of an exponential attraction between the interfaces. Strictly speaking the inverse length scale $\kappa$ now corresponds to the inverse bulk correlation length of the gas phase (or down-spin phase in a magnetic context), but for simplicity we shall assume an Ising-like symmetry for the bulk phases. The form of $\Delta W\left(\ell_{1}, \ell_{2}\right)$ may be easily derived from an underlying square-gradient DFT (or LGW Hamiltonian) by considering the excess free-energy of a constrained interfacial configuration. We will comment more on this later when we consider a non-local treatment of interfacial interactions.

Since, at equilibrium, the wetting films have the same thickness at each wall we can define $W(\ell ; L) \equiv W_{L}(\ell, L-\ell)$ and determine $W_{\text {sing }}$ as the minimum of

$$
W(\ell ; L)=2\left(\bar{h} \ell+a e^{-\kappa \ell}\right)-c e^{-\kappa(L-2 \ell)}+\cdots
$$

Provided the bulk ordering field is not too small, the interaction between the wetting films has a negligible influence on the equilibrium thickness which is extremely well approximated by the semi-infinite expression (11). This is certainly the case close to capillary condensation where, according to the Kelvin equation the film thickness $\ell \approx \xi_{\mathrm{b}} \ln L \ll L$. We note in passing that the interaction term does become important for much smaller values of $\delta \mu$ and, indeed, eventually induces a spinodal when $\bar{h} \approx e^{-2 \kappa L / 3}$ corresponding to thick wetting films of order $\ell \approx L / 3$. However, this metastable region is of no relevance to our present discussion of correlation functions near capillary condensation. It follows that near capillary condensation $W_{\text {sing }} \approx 2 \bar{h} \ell_{\pi}\left(\delta \mu_{\mathrm{co}}\right)$, so that equating (13) and (14) leads to

$$
\bar{h}_{\text {cap }}=\frac{2 \sigma}{\left(L-2 \ell_{\pi}\right)}+\cdots
$$

which is the modified Kelvin equation for complete wetting with short-ranged forces.
We now return our attention to our main concern and introduce the connected density-density correlation function $G\left(\mathbf{r}_{1}, \mathbf{r}_{2}\right)=\left\langle\delta \rho\left(\mathbf{r}_{1}\right) \delta \rho\left(\mathbf{r}_{2}\right)\right\rangle$ where $\delta \rho(\mathbf{r})=\rho(\mathbf{r})-\langle\rho(\mathbf{r})\rangle$ is the fluctuation (operator). It is convenient to exploit the translational invariance in the directions parallel to the wall and define a transverse or parallel Fourier transform

$$
\tilde{G}\left(z_{1}, z_{2} ; q\right) \equiv \int \mathrm{d} \mathbf{x}_{12} G\left(\mathbf{r}_{1}, \mathbf{r}_{2}\right) e^{i \mathbf{q} \cdot \mathbf{x}_{12}}
$$

where $\mathbf{x}_{12}$ is the parallel displacement vector between the two points at heights $z_{1}$ and $z_{2}$ from one of the walls. As a starting point for interpreting the correlation function we first consider the zeroth moment $\tilde{G}_{0}\left(z_{1}, z_{2}\right) \equiv \tilde{G}\left(z_{1}, z_{2} ; 0\right)$ corresponding to the zero wave-number limit. Indeed, exact sum-rules and also density-functional calculations shows that, for particle positions on either side of the slit, this function is related formally to the derivative of the total surface tension via [25]

$$
\tilde{G}_{0}(0, L) \propto-\frac{\partial^{2} \sigma(L)}{\partial L^{2}} .
$$

Therefore, for the vapour-like phase, provided $\delta \mu \geq \delta \mu_{\text {cap }}$, we can expect that

$$
\tilde{G}_{0}^{v}(0, L) \propto e^{-\kappa\left(L-2 \ell_{\pi}\right)} .
$$

As with the modified Kelvin equation, this expression depends on the reduced effective slit width $L-2 \ell_{\pi}$. Substituting for $\ell_{\pi}$, we have

$$
\tilde{G}_{0}^{v}(0, L) \propto \frac{e^{-\kappa L}}{\bar{h}^{2}}
$$

so that, exactly at capillary condensation,

$$
\tilde{G}_{0}^{v}(0, L) \propto L^{2} e^{-\kappa L}
$$

This result is notable because of the polynomial prefactor $\propto$ $L^{2}$ (arising from the reduction in the effective slit width) and contrasts with the expected result for the liquid-like phase

$$
\tilde{G}_{0}^{l}(0, L) \propto e^{-\kappa L} .
$$

Therefore correlations are enhanced due to the presence of wetting films. Note that the last expression (25) has two consistent interpretations. Firstly, model calculations for systems with short-ranged forces show that for the liquid-like phase the grand-potential (14) contains higher-order exponential corrections $\propto e^{-\kappa L}$ which lead directly to (25) using the sum-rule (21). More physically we know that away from the immediate vicinity of the walls where there are packing effects, the density profile is almost constant, in the liquid-like phase. Hence, we can anticipate that the pair-correlation function decays as it does in the bulk, provided of course that we are far below the capillary critical temperature. In other words, above the Fisher-Widom line, where the correlation function and associated inhomogeneous density profiles decay monotonically [26, 27], we anticipate a simple OrnsteinZernike-like decay

$$
G^{l}\left(\mathbf{r}_{1}, \mathbf{r}_{2}\right) \propto \frac{e^{-\kappa r}}{r}
$$


where $r \equiv\left|\mathbf{r}_{1}-\mathbf{r}_{2}\right|$ is the particle separation. The zeroth parallel moment of this, $\tilde{G}_{0} \propto e^{-\kappa z}$, decays as a pure exponential, so that, for particles on either side of the slit, we can expect (25). We emphasize again that the predictions (24) and (25) are only valid far below the capillary critical point. For wide capillary slits, this is equivalent to the condition that $L \gg \xi_{\mathrm{b}}$.

\section{B. Capillary-wave theory}

Let us now extend the analysis to calculate the paircorrelation function across the slit in the presence of wetting films assuming that $\delta \mu \geq \delta \mu_{\text {cap }}$. The simplest approach to this is based on a local interfacial Hamiltonian

$$
\mathcal{H}\left[\ell_{1}, \ell_{2}\right]=\int \mathrm{d} \mathbf{x}\left\{\frac{\sigma}{2}\left(\nabla \ell_{1}\right)^{2}+\frac{\sigma}{2}\left(\nabla \ell_{2}\right)^{2}+W_{L}\left(\ell_{1}, \ell_{2}\right)\right\}
$$

in which fluctuations are resisted by the surface tension and the interface interaction is modelled using the binding potential (16) with the expressions (10) and (17). To determine $G$ for particle positions close to the walls on opposite sides of the slit we need to determine the height-height correlations between the two different interfaces. To this end we define a $(2 \times 2)$ matrix $\tilde{\mathbf{S}}(q)$ with elements

$$
\tilde{S}_{m n}(q) \equiv \int \mathrm{d} \mathbf{x}_{12}\left\langle\delta \ell_{m}\left(\mathbf{x}_{1}\right) \delta \ell_{n}\left(\mathbf{x}_{2}\right)\right\rangle e^{i \mathbf{q} \cdot \mathbf{x}_{12}}
$$

where $\delta \ell_{m}(\mathbf{x})=\ell_{m}(\mathbf{x})-\left\langle\ell_{m}\right\rangle$. Recall that only two elements are independent since by symmetry $\tilde{S}_{11}=\tilde{S}_{22}$ and $\tilde{S}_{12}=$ $\tilde{S}_{21}$. At mean-field level, the matrix $\tilde{\mathbf{S}}(q)$ is obtained as the inverse of the matrix

$$
\mathbf{C}(q)=\beta\left(\begin{array}{cc}
\frac{\partial^{2} W_{L}}{\partial \ell_{1}^{2}}+\sigma q^{2} & \frac{\partial^{2} W_{L}}{\partial \ell_{1} \partial \ell_{2}} \\
\frac{\partial^{2} W_{L}}{\partial \ell_{2} \partial \ell_{1}} & \frac{\partial^{2} W_{L}}{\partial \ell_{2}^{2}}+\sigma q^{2}
\end{array}\right)
$$

the elements of which are simply the Fourier transforms of the second functional derivatives $\frac{\delta^{2} \mathcal{H}}{\delta \ell_{m} \delta \ell_{n}}$ evaluated at equilibrium.

Close to capillary condensation, $\tilde{C}_{11}(q)$ is essentially unchanged from the expression (6) for complete wetting in the semi-infinite geometry, while for the off-diagonal element is determined as the second derivative of $\Delta W\left(\ell_{1}, \ell_{2}\right)$. Thus,

$$
\mathbf{C}(q) \approx \beta\left(\begin{array}{cc}
W^{\prime \prime}\left(\ell_{\pi}\right)+\sigma q^{2} & -\kappa^{2} c e^{-\kappa\left(L-2 \ell_{\pi}\right)} \\
-\kappa^{2} c e^{-\kappa\left(L-2 \ell_{\pi}\right)} & W^{\prime \prime}\left(\ell_{\pi}\right)+\sigma q^{2}
\end{array}\right)
$$

This leads to the two structure factors

$$
\beta \tilde{S}_{11}(q) \approx \frac{1}{\kappa \bar{h}+\sigma q^{2}}
$$

and

$$
\beta \tilde{S}_{12}(q) \approx \frac{c \kappa^{2} e^{-\kappa\left(L-2 \ell_{\pi}\right)}}{\left(\kappa \bar{h}+\sigma q^{2}\right)^{2}}
$$

The first result is the same expression (or rather very nearly the same) as the structure factor for complete wetting at a single wall, eq. (7). The weak correlation between the two interfaces is quantified by the off-diagonal term $\tilde{S}_{12}$ which is exponentially small in the slit width. Note also that the denominator is the square of that appearing in $\tilde{S}_{11}(q)$.

To determine the density-density correlation function we note, that in local effective Hamiltonian theories, a local fluctuation in the interfacial height $\ell_{1}(\mathbf{x})$ produces an analogous local density fluctuation at the wall $\delta \rho \approx e^{-\kappa \ell_{1}} \delta \ell_{1}(\mathbf{x})$. This can be thought of as arising from the tail of the exponentially decaying density profile from the liquid-vapour interface. It follows that the Fourier transform $\tilde{G}(0, L ; q)$ of the densitydensity correlation function for particle positions on either side of the slit is given by

$$
\tilde{G}^{\mathrm{v}}(0, L ; q) \propto \frac{e^{-\kappa L}}{\left(\kappa \bar{h}+\sigma q^{2}\right)^{2}} .
$$

Exactly at capillary condensation, this reads

$$
\tilde{G}^{\mathrm{v}}(0, L ; q) \propto \frac{L^{2} e^{-\kappa L}}{\left(1+\frac{L}{2 \kappa} q^{2}\right)^{2}}
$$

Setting $q=0$, this recovers our earlier sum-rule result for the zeroth moment (24) demonstrating the consistency of the calculation. Note that the large length scale present in the wavevector dependence is simply the parallel correlation length $\xi_{\|} \propto \bar{h}^{-1 / 2}$ describing the interfacial fluctuations in the wetting film evaluated at the chemical potential corresponding to capillary condensation. Substituting the Kelvin equation expression $\delta \mu \approx 2 \sigma /(\Delta \rho L)$ into (12) yields

$$
\xi_{\|}=\sqrt{\frac{L}{2 \kappa}}
$$

which is the same as that appearing in (34).

Finally we need to perform the inverse Fourier transform to determine the desired decay in real space:

$$
G^{\mathrm{v}}(0, L)=C \frac{L^{2} e^{-\kappa L}}{2 \pi} \int_{0}^{\Lambda} \mathrm{d} q \frac{q}{\left(1+\frac{L}{2 \kappa} q^{2}\right)^{2}}
$$

where $C$ is an unimportant constant. It is interesting that this result is essentially independent of the cut-off $\Lambda$ because the high momentum behaviour is integrable. One can see this explicitly if one introduces a simple change of variable to rewrite the expression as

$$
G^{\mathrm{v}}(0, L)=C \frac{L e^{-\kappa L}}{2 \pi \xi_{b}} I_{C W}
$$

where

$$
I_{\mathrm{CW}}=\int_{0}^{\Lambda^{2} \xi_{b} L / 2} \mathrm{~d} z \frac{1}{(1+z)^{2}}
$$

is a dimensionless integral. Obviously this is non-singular and approaches unity in the limit $L \rightarrow \infty$. Thus within the local effective Hamiltonian theory we are led to the prediction

$$
\text { URL: http://mc.manuscriptcentral.com/tandf(trn }(\hat{\rho}, h L)=C \frac{L e^{-\kappa L}}{2 \pi \xi_{b}}
$$


which shows explicitly the enhancement of correlations across the slit compared to correlations in the condensed, liquid phase. The non-universal constant $C$ is the same as that appearing in the zeroth moment, and can be eliminated by defining a dimensionless amplitude ratio $L \xi_{b} G^{\mathrm{v}}(0, L) / \tilde{G}_{0}^{\mathrm{v}}(0, L)$ or, more accurately, the limit of this as $L \rightarrow \infty$. Thus, according to the present local Hamiltonian, this dimensionless amplitude ratio is given by

$$
\xi_{b} L \frac{G^{\mathrm{v}}(0, L)}{\tilde{G}_{0}^{\mathrm{v}}(0, L)} \approx \frac{1}{2 \pi}
$$

the value of which we shall return to shortly.

\section{Non-local theory}

The analysis described above, based on a local interfacial Hamiltonian, is reliable regarding most of its predictions. However, when we compare it to the results of a more microscopic DFT we find that it is not quite correct (see Appendix). In this section, we show that this can be traced directly to non-local effects associated with the damping of interfacial interactions and has a similar interpretation to recent studies of non-locality at short-ranged wetting transitions [28-31].

One may improve on the basic phenomenological interfacial Hamiltonian of wetting by starting from a more microscopic LGW theory and integrating out degrees of freedom systematically. This has been done recently for (short-ranged) wetting in a semi-infinite geometry and has provided new insights into a number of long-standing problems in the theory of critical wetting transitions [32]. We recall some details of this approach, concentrating only on what we need for complete wetting transitions, before describing how the analysis generalises to the parallel-plate geometry. The systematic derivation of an interfacial model was first considered by Fisher and Jin [33] who used a perturbative approach (as an expansion in the gradient $\nabla \ell$ ). However as described in [29], it is possible to do this non-perturbatively using a Green's function method closely related to multiple reflection expansion techniques [34]. This has a number of advantages and allows one to derive an interfacial model for wetting at nonplanar walls, the position of which we denote by a function $\psi(\mathbf{x})$.

The non-local (NL) Hamiltonian can be written

$$
\mathcal{H}_{\mathrm{NL}}[\ell, \psi]=\sigma A_{\mathrm{lv}}+\bar{h} V_{\ell}+W_{\mathrm{NL}}[\ell, \psi]
$$

and is valid for length scales larger than the bulk correlation length. Curvature corrections related to rigidity-like terms can be calculated but are not considered here. The interfacial area is denoted $A_{\mathrm{lv}}$ while $V_{\ell}=\int d \mathbf{x}(\ell(\mathbf{x})-\psi(\mathbf{x}))$ is the volume of the wetting film. The binding potential functional $W_{\mathrm{NL}}[\ell, \psi]$ describes the interaction of the interface and wall and has an expansion

$$
W_{N L}[\ell, \psi]=a \Omega_{1}^{1}[\ell, \psi]+\cdots
$$

with a geometry independent coefficient $a$ which is the same as the binding potential (10). Higher order contributions to
$W_{\mathrm{NL}}$ exist but are not important for complete wetting transitions [35]. Each of these contributions has a diagrammatic representation in which a zigzagging straight line (each zig and zag representing a kernel ) connects points on the wall (lower wavy-line) to points on the interface (upper wavy-line). The dominant diagram has the algebraic expression

$$
\Omega_{1}^{1}[\ell, \psi]=\widetilde{\mathcal{C}}=\iint \mathrm{d} \mathbf{s}_{\psi} \mathrm{d} \mathbf{s}_{\ell} K\left(\mathbf{r}_{\psi}, \mathbf{r}_{\ell}\right)
$$

Here $\mathbf{r}_{\psi}$ and $\mathbf{r}_{\ell}$ are points on the wall and interface respectively, which are integrated over using the appropriate infinitesimal areas $\mathrm{d} \mathbf{s}_{\psi}=\mathrm{d} \mathbf{x} \sqrt{1+(\nabla \psi)^{2}}$ and $\mathrm{d} \mathbf{s}_{\ell}=$ $\mathrm{d} \mathbf{x} \sqrt{1+(\nabla \ell)^{2}}$. These surface integrations are implied by the black circles in the diagrams. The kernel, denoted by each straight line, is

$$
K\left(\mathbf{r}_{1}, \mathbf{r}_{2}\right)=\frac{\kappa}{2 \pi\left|\mathbf{r}_{1}-\mathbf{r}_{2}\right|} e^{-\kappa\left|\mathbf{r}_{1}-\mathbf{r}_{2}\right|}
$$

and is simply a normalised version of the bulk correlation function.

Specialising to the case of wetting at a planar wall, the NL Hamiltonian reduces to

$$
\mathcal{H}_{\mathrm{NL}}[\ell]=\int \mathrm{d} \mathbf{x}\left\{\frac{\sigma}{2}(\nabla \ell)^{2}+\bar{h} \ell\right\}+W_{\mathrm{NL}}[\ell]
$$

where we have taken the usual gradient expansion for the bending term. If the wall is flat the integral over it can be done exactly in which case the wetting diagram reduces to

$$
\widetilde{\gamma}=\int \mathrm{d} \mathbf{s} e^{-\kappa \ell}
$$

where $\mathrm{d} \mathbf{s}=\mathrm{d} \mathbf{x} \sqrt{1+(\nabla \ell)^{2}}$. One can see immediately that this contribution is essentially identical to that appearing in the standard interfacial Hamiltonian arising from the (leading) exponential decay of the binding potential (10). That is, for a planar wall, the leading-order contribution to the binding potential functional is local in character. This is not the case for higher order terms, which are important at critical wetting transitions, but for complete wetting the form of the local Hamiltonian (3) is supported by the NL theory. Consequently the predictions for the parallel correlation $\xi_{\|}$and structure factor $\tilde{S}(q)$ are the same as described in section III A.

Despite this the description of density-density correlation functions is better within the NL theory because the relation between fluctuations in the interfacial height and density fluctuations is controlled explicitly by the kernel $K$. Indeed this is an additional source of non-local effects with the result that the NL description is in precise agreement with an exact sumrule [31]. The singular (interfacial) contribution to the pair correlation function is given by

$$
G\left(\mathbf{r}_{1}, \mathbf{r}_{2}\right) \propto \frac{\partial^{2}}{\partial z_{1} \partial z_{2}} \iint \mathrm{d} \mathbf{x}_{3} \mathrm{~d} \mathbf{x}_{4} K\left(\mathbf{r}_{1}, \mathbf{r}_{3}\right) S\left(x_{34}\right) K\left(\mathbf{r}_{4}, \mathbf{r}_{2}\right)
$$


where $\mathbf{r}_{1}$ and $\mathbf{r}_{2}$ are the correlated points, while $\mathbf{r}_{3}=\left(\mathbf{x}_{3}, \ell_{\pi}\right)$ and $\mathbf{r}_{4}=\left(\mathbf{x}_{4}, \ell_{\pi}\right)$ lie on the interface. This has the short-hand diagrammatic representation

$$
G\left(\mathbf{r}_{1}, \mathbf{r}_{2}\right) \propto \frac{\partial^{2}}{\partial z_{1} \partial z_{2}} \text { ? }
$$

where the wiggly line represents the height-height correlation function $S\left(x_{12}\right)$. In Fourier space, this reads

$$
\tilde{G}\left(z_{1}, z_{2} ; q\right) \propto \tilde{K}^{\prime}\left(z_{1}-\ell_{\pi} ; q\right) \tilde{K}^{\prime}\left(z_{2}-\ell_{\pi} ; q\right) \tilde{S}(q)
$$

where

$$
\tilde{K}(z ; q)=\frac{\kappa}{\kappa_{q}} e^{-\kappa_{q}|z|}
$$

is the parallel Fourier transform of the Green's function (44), the prime denotes the derivative w.r.t. $z$ and we have abbreviated $\kappa_{q}=\sqrt{\kappa^{2}+q^{2}}$. Note that the length scale controlling the exponential decay of correlations as we move away from the interface is $\kappa_{q}$ and not $\kappa$ (as is often assumed in local theories). Thus for particle positions very close to the wall, the NL theory predicts that $\tilde{G}$ contains a singular term

$$
\tilde{G}_{\text {sing }}(0,0 ; q) \propto \frac{\bar{h}}{1+\xi_{\|}^{2} q^{2}} e^{-\ell_{\pi} q^{2} / \kappa} .
$$

Note the presence of a damping factor $e^{-\ell_{\pi} q^{2} / \kappa}$ which shows that, in addition to $\xi_{\|}$, there is a diverging length scale $\xi_{\mathrm{NL}} \propto$ $\sqrt{\ell_{\pi}}$ which strongly damps correlations. It is the allowance for this length in the NL theory which gives consistency with the exact sum-rule requirement that the second moment $\tilde{G}_{2}(0,0)_{\text {sing }} \propto \sigma+\sigma_{\text {sing }}[30,31]$.

The generalisation of the NL theory to wetting films in a parallel-slit geometry is straightforward. The interaction of each interface with the (closest) wall is the same as in the semi-infinite geometry and is essentially local in character. The difference with the local Hamiltonian approach however is that the interaction between the interfaces is now strongly non-local. Thus, we have to write

$$
\mathcal{H}_{\mathrm{NL}}\left[\ell_{1}, \ell_{2}\right]=\int \mathrm{d} \mathbf{x}\left\{\frac{\sigma}{2}\left(\nabla \ell_{1}\right)^{2}+\frac{\sigma}{2}\left(\nabla \ell_{2}\right)^{2}\right\}+W_{L}\left[\ell_{1}, \ell_{2}\right]
$$

where

$$
W_{L}\left[\ell_{1}, \ell_{2}\right] \equiv \int \mathrm{d} \mathbf{x}\left\{W\left(\ell_{1}\right)+W\left(L-\ell_{2}\right)\right\}+\Delta W\left[\ell_{1}, \ell_{2}\right]
$$

analogous to (16). The interaction between the interfaces is now modelled by the non-local expression

$$
\Delta W\left[\ell_{1}, \ell_{2}\right] \equiv-c \Omega_{1}^{1}\left[\ell_{1}, \ell_{2}\right]
$$

where, in an obvious notation,

$$
\Omega_{1}^{1}\left[\ell_{1}, \ell_{2}\right] \equiv \iint \mathrm{d} \mathbf{s}_{\ell_{1}} \mathrm{~d} \mathbf{s}_{\ell_{2}} K\left(\mathbf{r}_{\ell_{1}}, \mathbf{r}_{\ell_{2}}\right)
$$

Again, we can visualise this interaction diagrammatically as

$$
\Omega_{1}^{1}\left[\ell_{1}, \ell_{2}\right] \equiv \overline{\underline{\underline{Z}}}
$$

where the two horizontal lines represent the capillary walls. If the two interfaces are planar then this integral reduces to

$$
\overline{\check{C}}=A e^{-\kappa\left(\ell_{2}-\ell_{1}\right)}
$$

where $A$ is the area. This exactly reproduces the interaction term (17) in the binding potential function (16) implying that, for planar interfaces, the NL Hamiltonian is (per unit area) identical to the binding potential function $W_{L}\left(\ell_{1}, \ell_{2}\right)$ appearing in the local theory. Hence, at mean-field level, the excess free-energy and therefore the zeroth moment of the pair correlation function $\tilde{G}_{0}(0, L)$ are the same as calculated earlier.

The determination of the structure factor $\tilde{S}_{12}(q)$ follows along the same lines described in III A. The difference between the local and NL theories arises because now the offdiagonal terms for the direct correlation function matrix is wave-number dependent

$$
\tilde{C}_{12}(q) \approx-\kappa^{2} c e^{-\kappa_{q}\left(L-2 \ell_{\pi}\right)}
$$

and recall $\kappa_{q}=\sqrt{\kappa^{2}+q^{2}}$. This feeds directly into the structure factor

$$
\beta \tilde{S}_{12}(q) \approx \frac{c \kappa^{2} e^{-\kappa_{q}\left(L-2 \ell_{\pi}\right)}}{\left(\kappa \bar{h}+\sigma q^{2}\right)^{2}}
$$

which only matches with the corresponding expression of the local theory when $q=0$. To calculate the pair-correlation function, we use (49) which is equivalent to

$$
\tilde{G}^{\mathrm{v}}(0, L ; q) \propto e^{-2 \kappa_{q} \ell_{\pi}} \tilde{S}_{12}(q)
$$

where we have assumed that the wetting layer thickness is the same as at a single wall. Hence, for $\bar{h} \geq \bar{h}_{\text {cap }}$, we have

$$
\tilde{G}^{\mathrm{v}}(0, L ; q) \propto \frac{e^{-\sqrt{\kappa^{2}+q^{2}} L}}{\left(\kappa \bar{h}+\sigma q^{2}\right)^{2}} .
$$

This is similar, but not quite identical, to the result (34) we arrived at using a local capillary-wave theory because of the wave-vector dependence in the numerator. Since the NL Hamiltonian is only valid for length scales longer than the bulk correlation length we can expand $\kappa_{q} \approx \kappa+q^{2} / 2 \kappa+\cdots$ and re-write this as

$$
\tilde{G}^{\mathrm{v}}(0, L ; q) \propto e^{-\kappa L} \frac{e^{-q^{2} L \xi_{b} / 2}}{\left(\kappa \bar{h}+\sigma q^{2}\right)^{2}}
$$

which identifies an additional damping term in the numerator $\propto e^{-L q^{2} \xi_{b} / 2}$ compared with the corresponding result of the local theory. Thus both approaches are in agreement with regard to the value of the zeroth moment but differ for larger 
wave-vectors. It is apparent that the structure factor across the slit is characterised by two large length scales: the usual parallel correlation length $\xi_{\|}$which, for $\bar{h} \geq \bar{h}_{\text {cap }}$, is well approximated by the semi-infinite result (12), and a second length

$$
\xi_{\text {damp }}=\sqrt{\frac{L \xi_{b}}{2}}
$$

which emerges from the damping term $e^{-L q^{2} \xi_{b} / 2} \equiv e^{-q^{2} \xi_{\text {damp }}^{2}}$. It is clear that this damping effect is very similar to that which occurs for the correlation function $\tilde{G}(0,0 ; q)$ at complete wetting discussed earlier. The extra length scale controlling the damping in this case was $\xi_{\mathrm{NL}}=\sqrt{\ell_{\pi} \xi_{b}}$. The damping across the capillary is similar but now occurs on the scale of the slit width rather than the wetting film thickness.

A remarkable feature of the length scales $\xi_{\text {damp }}$ and $\xi_{\|}$is that at capillary condensation, $\bar{h}_{\text {cap }} \approx 2 \sigma / L$, they are equal. In other words the Kelvin equation (1) is equivalent to the statement that

$$
\xi_{\text {damp }}=\xi_{\|}
$$

where $\xi_{\|}$is given by (12). Expressed in terms of the slit width, we find for the Fourier transform

$$
\tilde{G}^{\mathrm{v}}(0, L ; q) \approx C L^{2} e^{-\kappa L} \frac{e^{-L q^{2} / 2 \kappa}}{\left(1+\frac{L}{2 \kappa} q^{2}\right)^{2}}
$$

which is valid for wave-numbers $q \ll \Lambda \approx \kappa$, and $L \gg \xi_{\mathrm{b}}$. The scaling of the correlation lengths (64) therefore implies that

$$
G^{\mathrm{v}}(0, L)=C \frac{L e^{-\kappa L}}{2 \pi \kappa} I_{\mathrm{NL}}
$$

where

$$
I_{\mathrm{NL}}=\int_{0}^{\Lambda^{2} \xi_{b} L / 2} \mathrm{~d} z \frac{e^{-z}}{(1+z)^{2}}
$$

is different to the corresponding expression $I_{\mathrm{CW}}$ of the local theory. Again this integral is very weakly dependent on $L$ (which can be set to $\infty$ ) leading to

$$
I_{\mathrm{NL}}=1-e E_{1}(1) \approx 0.404
$$

where $E_{1}(x)$ is the exponential integral

$$
E_{1}(x) \equiv \int_{x}^{\infty} d t \frac{e^{-t}}{t}
$$

Hence the dimensionless amplitude ratio

$$
\xi_{b} L \frac{G^{\mathrm{v}}(0, L)}{\tilde{G}_{0}^{\mathrm{v}}(0, L)} \approx \frac{1-e E_{1}(1)}{2 \pi}
$$

and is reduced compared to the prediction of the local interfacial Hamiltonian. In the Appendix, we show that (65) is the result obtained from directly solving the Ornstein-Zernike equation for a square gradient DFT. That is, only the nonlocal description of interfacial interactions reproduces the full quantitative features of an underlying microscopic theory.
Finally, we mention that within the non-local theory there is a simple diagrammatic expression for the correlation function across the slit, analogous to that for a single wall, eq. (48). For particle positions at distances $z_{1} \approx 0$ and $z_{2} \approx L$ (and arbitrary parallel displacement), the expression is given by

$$
G\left(\mathbf{r}_{1}, \mathbf{r}_{2}\right) \propto \frac{\partial^{2}}{\partial z_{1} \partial z_{2}} \stackrel{\boldsymbol{\rho}}{\longrightarrow}
$$

where the lowest and highest horizontal lines represent the capillary walls, and the two middle horizontal lines represent the two liquid-vapour interfaces. The diagram has the same interpretation as earlier, in which the wavy lines denote height-height correlations $S_{11}\left(=S_{22}\right)$, and the thick line denotes the kernel $K$. In this way, we can see rather clearly that the total correlation across the slit arises from both bulk-like decays, represented by the thick lines, and capillary-wave excitations at both interfaces (wavy lines).

\section{DISCUSSION}

In this paper, we have discussed how the presence of wetting films influences the density (or magnetisation) correlation function $G(0, L)$ between particle positions on either side of a capillary slit (or Ising thin-film). Concentrating on systems in three dimensions with short-ranged forces, our central predictions are as follows:

1) The amplitude of the exponential decay of correlations is enhanced due to the presence of the wetting films. Near capillary condensation, the correlation function in the vapour phase decays as $G^{\mathrm{v}}(0, L) \approx L e^{-\kappa L}$ compared to $G^{\mathrm{l}}(0, L) \approx$ $e^{-\kappa L} / L$ in the liquid phase.

2) The enhancement of correlations arises from the interaction between the fluctuating interfaces on either side of the slit and, unlike the interfacial roughness, does not depend on the high-momentum cut-off in the effective Hamiltonian.

3) The attractive interaction between the wetting films must be modelled using a non-local interaction potential which we can represent diagrammatically as

$$
\Delta W\left[\ell_{1}, \ell_{2}\right]=-c \overline{\widetilde{\alpha}} .
$$

For planar interfacial configurations this recovers the usual binding potential function present in local descriptions. This non-local description correctly accounts for the damping of interfacial interactions controlled by a length scale

$$
\xi_{\text {damp }}=\sqrt{\frac{L \xi_{b}}{2}}
$$

4) At capillary condensation $\bar{h}_{\text {cap }} \approx 2 \sigma / L$ the damping length scale and the parallel correlation length are equal:

$$
\xi_{\text {damp }}=\xi_{\|}
$$


This scaling of the characteristic lengths leads to the following amplitude ratio for the correlation function to its zeroth moment

$$
\xi_{b} L \frac{G^{\mathrm{v}}(0, L)}{\tilde{G}_{0}^{\mathrm{v}}(0, L)} \approx \frac{1-e E_{1}(1)}{2 \pi} .
$$

This may be regarded as a signature of non-locality.

Our analysis has only considered Gaussian fluctuations about the mean-field approximation. However, we believe that our quantitative predictions remain valid even if one did a more thorough renormalisation group analysis based on the interfacial models. As remarked earlier, even though the upper critical dimension for complete wetting with short-ranged forces is $d^{*}=3$, it is known that fluctuations do not alter the mean-field expression for the parallel correlation length (12). Since both the form of the exponential decay of correlations and the expression for $\xi_{\|}$are not altered by interfacial fluctuation effects in three dimensions, we believe all our predictions are exact (for sufficiently large slit widths $L$ ).

We finish our article by making some brief remarks about systems with long-ranged forces and also correlation functions near (pseudo) capillary condensation in two dimensions:

Long-Ranged Forces - Let us imagine that instead of being short-ranged, the attractive component of the fluid-fluid (and wall-fluid) intermolecular potential decays as $\phi(r) \approx-r^{-\gamma}$ where, of course, $\gamma=6$ for dispersion forces. In the bulk, this long-ranged decay, also describes the large-distance behaviour of the correlation function. It is possible to generalise the above interfacial Hamiltonian analysis and determine how the correlation function behaves in the slit geometry in the presence of wetting films. Consider a local treatment first using the interfacial Hamiltonian (27) but now with a binding potential appropriate for long-ranged forces. For the wallinterface interaction we use the standard binding potential [12-15]

$$
W(\ell)=\bar{h} \ell+a \ell^{-p}+\cdots
$$

where $p=\gamma-4$ (in three dimensions). To describe the interinterfacial interaction we now use

$$
\Delta W\left(\ell_{2}, \ell_{1}\right) \propto\left(\ell_{2}-\ell_{1}\right)^{-p}+\cdots
$$

which is simply the excess free-energy per unit area of two planar parallel interfaces separated by a distance $\ell_{2}-\ell_{1}$.

Repeating our analysis, we find that this local treatment predicts that, at capillary condensation, the correlation decays as $G(0, L) \sim L^{-q}$ where the exponent $q=\frac{(\gamma-2)^{2}}{\gamma-3}$, i.e., an algebraic decay which is different to the intermolecular potential! For example, $q=16 / 3<6$ for dispersion forces. This result is, in fact, wrong owing to the incorrect local description of the interface-interface interaction. Recall that, for simple liquids, it is often assumed that the contribution to the Helmholtz functional $F[\rho]$, arising from attractive intermolecular forces is

$$
F_{\text {att }}=\frac{1}{2} \iint \mathrm{d} \mathbf{r}_{1} \mathrm{~d} \mathbf{r}_{2} \rho\left(\mathbf{r}_{1}\right) \rho\left(\mathbf{r}_{2}\right) \phi\left(r_{12}\right)
$$

Applying a simple sharp-kink approximation to the density profile in this expression leads directly to an interaction term

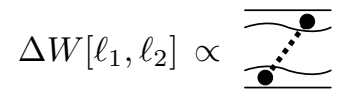

where, following the diagrammatic notation of [35], the upper and lower black dots (which now lie off a surface) indicate an integral over the volume of the upper and lower wetting layer. The dashed line now represents the intermolecular potential $\phi(r)$ rather than the Yukawa function $K(r)$. If both $\ell_{1}$ and $\ell_{2}$ are planar $\Delta W\left[\ell_{1}, \ell_{2}\right]=A \Delta W\left(\ell_{1}, \ell_{2}\right)$ (where $A$ is the area), so that the local theory is recovered in the long-wavelength limit. It is straightforward to show that the non-local interaction (79) corrects the erroneous prediction of the local theory and leads to

$$
G(0, L) \approx L^{-\gamma}
$$

i.e., the pair correlation decays algebraically with the same exponent as the intermolecular potential. Wetting films therefore do not strongly influence the decay of correlations as they do for systems with short-ranged forces.

Two-Dimensional Systems - Finally, we mention that correlation functions at two-dimensional capillary condensation are also of interest. Consider, for example, a two dimensional Ising strip of infinite length, finite width $L$ and surface spins fixed to +1 (infinite surface field). Since the system is only infinite in one direction, it is pseudo-one dimensional and the capillary condensation "transition" is rounded. However, for wide slits, the cross-over from up-spin to down-spin phases is sharp and occurs over an exponentially narrow region centred around $\bar{h}_{\text {cap }}$ [36]. It is still possible, therefore, to compare how the correlation function between spins on either side of the slit decays compared to that in the bulk.

Recall that exactly in zero bulk field, and at sub-critical temperatures, the spin-spin correlation function decays as $G(r) \approx \mathrm{e}^{-\kappa r} / r^{2}$ where the inverse length scale is related to the surface tension $\kappa=2 \beta \sigma$ [37]. These features of the bulk correlation function are very beautifully explained by Abraham's bubble model of correlations [38]. In this picture, correlations in a phase of net down-spins (say) arise from bubbles of up-spins which enclose the two points being correlated. The sum over all Boltzmann weighted bubble configurations determines the correlation function $G(r) \propto \Sigma \mathrm{e}^{-\beta E}$. In Fig. 2 we schematically illustrate the lowest energy bubble excitation in the down-spin and up-spin-like phases close to condensation. For the down-spin phase, the bubble that connects spins on either side of the slit has the form of a capillary bridge made from two semi-circular menisci. For the upspin phase, on the other hand, a circular bubble of down-spins stretches across the slit connecting the two correlated spins. The energy cost of both these configurations is determined by simple macroscopic considerations involving surface tensions (conjugate to the length of the domain wall) and the coupling to the bulk magnetic field (conjugate to the area of the excitation). Precisely at capillary condensation the energy cost of 

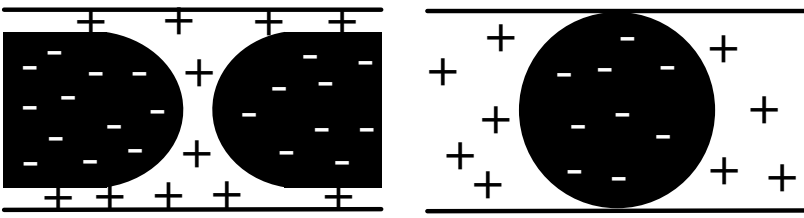

FIG. 2. In 2D systems, spins on either side of the strip are correlated when excitations corresponding to bubbles of the appropriate metastable phase extend between the two spins. Left) The elementary excitation on the down (-) spin phase is a capillary bridge of up $(+)$ spins. Right) The elementary excitation on the up (+) spin phase is a circular bubble of down spins.

both the bridge and circular bubble excitations are the same and a simple calculation leads to the prediction

$$
G(0, L) \approx e^{-\pi \kappa L / 4}
$$

for both the up-spin and down-spin phase. Here, we have ignored a possible multiplicative algebraic prefactor which depends on $L$ and is unimportant compared to the exponential term. It is remarkable therefore that near to capillary condensation the correlation function decays with an effective (inverse) length scale $\kappa_{\text {eff }}=\frac{\pi}{4} \kappa$. Since $\kappa_{\text {eff }}<\kappa$, it follows that correlations are therefore enhanced in the slit geometry. We believe this prediction can be checked numerically in the Ising model using density-matrix renormalisation group methods $[39,40]$ or perhaps simulations.

\section{ACKNOWLEDGMENTS}

It is our pleasure to dedicate this paper to Bob Evans on the occasion of his (unexpected) 65th birthday. Throughout his academic life, he has been nothing other than an inspiration. A.O.P. is particularly grateful for the wonderful memories of his time in Bristol.

NRB acknowledges support from the Portuguese Foundation for Science and Technology (SFRH/BPD/63183/2009). CR acknowledges support from grants MOSAICO (Ministerio de Educación y Ciencia) and MODELICO (Comunidad de Madrid).

\section{Appendix A: Correlation Functions within a Square-Gradient DFT}

In this appendix, we determine the pair-correlation function across a capillary slit using a simple square gradient DFT. We adopt a magnetic notation and consider the LGW free-energy functional

$$
\mathcal{F}[m]=\int \mathrm{d} \boldsymbol{r}\left\{\frac{1}{2}(\nabla m(\boldsymbol{r}))^{2}+\phi(m(\boldsymbol{r}))\right\}
$$

with two bounding walls in the $z=0$ and $z=L$ planes. The local order parameter (magnetization) is denoted $m(\boldsymbol{r})$, and the potential $\phi(m(\boldsymbol{r}))$ has an appropriate double-well structure modelling bulk two-phase coexistence at subcritical temperatures. We use the simplest possible "choice, corresponding $\tilde{G}$ satisfies to a double-parabola approximation

$$
\phi(m)=\frac{\kappa^{2}}{2}\left(|m|-m_{0}\right)^{2}-h m
$$

where the bulk "liquid" and "gas" phases have order parameters $m_{0}$ and $-m_{0}$ at zero field, respectively. We fix the magnetization at the walls to be $m_{1}$, and note that the condition $m_{1}>m_{0}$ corresponds to complete wetting, $\theta=0$. The equilibrium magnetization profile is determined by the EulerLagrange equation

$$
m^{\prime \prime}(z)=\phi^{\prime}(m)
$$

whose solution, for the double-parabola approximation, is readily expressed in terms of elementary exponential functions. The correlation function $G\left(\boldsymbol{r}_{1}, \boldsymbol{r}_{2}\right)=\left\langle m\left(\boldsymbol{r}_{1}\right) m\left(\boldsymbol{r}_{2}\right)\right\rangle-$ $\left\langle m\left(\boldsymbol{r}_{1}\right)\right\rangle\left\langle m\left(\boldsymbol{r}_{2}\right)\right\rangle$ is determined from solution of the OrnsteinZernike equation [41]. In Fourier space, the parallel transform

$$
\left[-\partial_{z_{1}}^{2}+\phi^{\prime \prime}\left(m\left(z_{1}\right)\right)+q^{2}\right] \tilde{G}\left(z_{1}, z_{2} ; q\right)=\delta\left(z_{1}-z_{2}\right)
$$

where we have set $\mathrm{k}_{\mathrm{B}} T=1$. Again within double-parabola approximation, this equation is readily solved since $\phi^{\prime \prime}(m)=$ $\kappa^{2}$, provided $m \neq 0$ [31]. For the "vapour-like" phase, the full solution for $G$ is

$$
\tilde{G}^{\mathrm{v}}\left(z_{1}, z_{2} ; q\right)=\frac{2 \kappa_{q} \sinh \left(\kappa_{q}\left(L-z_{2}\right)\right) \sinh \left(\kappa_{q} z_{1}\right) \mathrm{e}^{-\kappa_{q} L}}{\left(\frac{\kappa^{2} m_{0}}{\left|m^{\prime}(\ell)\right|}\right)^{2} X_{1}-\kappa_{q} \frac{2 \kappa^{2} m_{0}}{\left|m^{\prime}(\ell)\right|} X_{2}+\kappa_{q}^{2} X_{3}}
$$

where

$$
\begin{aligned}
& X_{1}=\left(1-\mathrm{e}^{-2 \kappa_{q}(L-\ell)}\right)\left(1-\mathrm{e}^{-2 \kappa_{q} \ell}\right)^{2} \\
& X_{2}=\left(1-\mathrm{e}^{-2 \kappa_{q}(L-\ell)}\right)\left(1-\mathrm{e}^{-2 \kappa_{q} \ell}\right) \\
& X_{3}=1-\mathrm{e}^{-2 \kappa_{q} L}
\end{aligned}
$$

Here, $\ell$ denotes the equilibrium position of the interface (where $m=0$ ) from the wall at $z=0$. We now consider the case of a very wide capillary slit, $L \gg l \gg 1 / \kappa$, and small magnetic fields, $|h| \ll \kappa^{2} m_{0}$. We can then approximate $X_{1} \approx X_{2} \approx X_{3} \approx 1$ and assume that the interface position is located in the same position as in the semi-infinite geometry. Also, setting $z_{1} \approx 1 / \kappa$ and $z_{2} \approx L-1 / \kappa$, the correlation function across the slit reduces to

$$
\begin{aligned}
& \tilde{G}^{\mathrm{v}}(0, L ; q) \approx \\
& \frac{\kappa_{q} \mathrm{e}^{-\kappa_{q} L} / 2}{\kappa^{2}\left(1-2 \kappa_{q} / \kappa\right)-\frac{2|h|}{m_{0}}\left(1-\kappa_{q} / \kappa\right)+\frac{|h|^{2}}{\kappa^{2} m_{0}^{2}}\left(3-2 \kappa_{q} / \kappa\right)+\kappa_{q}^{2}} .
\end{aligned}
$$

Expanding $\kappa_{q}$ to order $q^{4}$ in the denominator and $q^{2}$ in the numerator, this reduces to

$$
\tilde{G}^{\mathrm{v}}(0, L ; q) \approx 2 \kappa^{3} \sigma^{2} \mathrm{e}^{-\kappa L} \frac{\mathrm{e}^{-q^{2} L \xi_{\mathrm{b}} / 2}}{\left(2|h| m_{0} \kappa+\sigma q^{2}\right)^{2}} .
$$

where $\sigma \equiv \kappa m_{0}^{2}$ is the surface tension. 
[1] R. Evans, U. Marini Bettolo Marconi, and P. Tarazona, J. Chem. Phys. 84, 2376 (1986).

[2] B. V. Derjaguin and N. V. Churaev, in Fluid Interfacial Phenomena, edited by C. A. Croxton (Wiley, 1986) chapter 15 and references therein.

[3] M. Schoen, D. J. Diestler, and J. H. Cushman, J. Chem. Phys. 87, 5464 (NOV 1 1987).

[4] R. Evans, J. Phys.: Condens. Matter 2, 8989 (NOV 1990).

[5] L. Gelb, K. Gubbins, R. Radhakrishnan, and M. SliwinskaBartkowiak, Rep. Prog. Phys. 62, 1573 (DEC 1999).

[6] K. Binder, Ann. Rev. Mater. Res. 38, 123 (2008).

[7] W. F. Saam, J. Low Temp. Phys. 157, 77 (2009).

[8] H. Nakanishi and M. E. Fisher, J. Chem. Phys. 78, 3279 (1983).

[9] B. V. Derjaguin, Acta Physicochim. URSS 12, 181 (1940).

[10] A. O. Parry and R. Evans, J. Phys. A 25, 275 (JAN 1992).

[11] J. R. Henderson, Mol. Phys. 59, 89 (1986).

[12] S. Dietrich, in Phase Transitions and Critical Phenomena, Vol. 12, edited by C. Domb and J. Lebowitz (Academic Press Limited, 1988).

[13] M. Schick, in Liquids at interfaces, edited by J. Charvolin, J. F. Joanny, and J. Zinn-Justin (Elsevier, 1990).

[14] G. Forgacs, R. Lipowsky, and T. M. Nieuwenhuizen, in Phase Transitions and Critical Phenomena, Vol. 14, edited by C. Domb and J. Lebowitz (Academic Press Limited, 1991).

[15] D. Bonn, J. Eggers, J. Indekeu, J. Meunier, and E. Rolley, Rev. Mod. Phys. 81, 739 (APR-JUN 2009).

[16] H. Nakanishi and M. E. Fisher, Phys. Rev. Lett. 49, 1565 (1982).

[17] D. B. Abraham, Phys. Rev. Lett. 44, 1165 (1980).

[18] K. Binder, D. P. Landau, and D. M. Kroll, Phys. Rev. Lett. 56, 2272 (1986).

[19] A. N. Frumkin, Zh. Fiz. Khim. (J. Phys. Chem.) 12, 337 (1938).

[20] R. Roth, J. Phys.: Condens. Matter 22, 063102 (2010).

[21] M. E. Fisher and A. J. Jin, Phys. Rev. B 44, 1430 (1991).
[22] F. P. Buff, R. Lovett, and F. H. Stillinger, Phys. Rev. Lett. 15, 621 (1965).

[23] M. E. Fisher, J. Chem. Soc. 82, 1569 (1986).

[24] D. S. Fisher and D. A. Huse, Phys. Rev. B 32, 247 (1985).

[25] J. R. Henderson, in Fundamentals of Inhomogeneous Fluids, edited by D. Henderson (Marcel Dekker, Inc., 1992).

[26] M. E. Fisher and B. Widom, J. Chem. Phys. 50, 3756 (1969).

[27] R. Evans, J. R. Henderson, D. C. Hoyle, A. O. Parry, and Z. A. Sabeur, Mol. Phys. 80, 755 (1993).

[28] A. O. Parry, J. M. Romero-Enrique, and A. Lazarides, Phys. Rev. Lett. 93, 086104 (2004).

[29] A. O. Parry, C. Rascón, N. R. Bernardino, and J. M. RomeroEnrique, J. Phys.: Condens. Matter 18, 6433 (2006).

[30] A. O. Parry, C. Rascón, N. R. Bernardino, and J. M. RomeroEnrique, Phys. Rev. Lett. 100, 136105 (2008).

[31] A. O. Parry, J. M. Romero-Enrique, N. R. Bernardino, and C. Rascón, J. Phys.: Condens. Matter 20, 505102 (2008).

[32] A. O. Parry and C. Rascón, J. Low Temp. Phys. 157, 149 (2009).

[33] A. J. Jin and M. E. Fisher, Phys. Rev. B 47, 7365 (1993).

[34] R. Balian and C. Bloch, Ann. Phys. (NY) 60, 401 (1970).

[35] N. R. Bernardino, A. O. Parry, C. Rascón, and J. M. RomeroEnrique, J. Phys.: Condens. Matter 21, 465105 (2009).

[36] V. Privman and M. E. Fisher, J. Stat. Phys. 33, 385 (1983).

[37] L. Kadanoff, Nuovo Cimento 44, 276 (1966).

[38] D. B. Abraham, Phys. Rev. Lett. 50, 291 (1983).

[39] Density-Matrix Renormalization. A New Numerical Method in Physics, edited by I. Peschel, X. Wang, M. Kaulke, and K. Hallberg, Lecture Notes in Physics, Vol. 528 (Springer, Berlin, 1999).

[40] A. Drzewiński, A. Maciołek, A. Barasinski, and S. Dietrich, Phys. Rev. E 79, 041145 (2009).

[41] R. Evans, Adv. Phys. 28, 143 (1979). 